\title{
ENSAYOS DE CERTIFICACIÓN DE MÓDULOS FOTOVOLTAICOS
}

\author{
Dr. FIRMAN, Andrés ${ }^{(*)}$; Dr. VERA, Luis; Dr. CÁCERES, Manuel ${ }^{(*)}$; \\ Ing. GONZALEZ MAYANS, Alexis Raúl $\left.{ }^{*}\right) 3$; Sr. BERTOTTO, Olivio Augusto ${ }^{(*) 4}$
}

Palabras clave: Energía Solar fotovoltaica, instrumentación, caracterización eléctrica.

\section{Resumen}

Mediante el equipamiento de primera línea, adquirido gracias a la presentación en diversos proyectos en los que activamente participa, se logró desarrollar la capacidad de realizar ensayos de certificación de módulos fotovoltaicos (FV), según normas IRAM vigentes en el país. La exactitud conseguida en los ensayos para la potencia nominal es del 5,4 \% lo que es comparable a la de laboratorios de otras regiones o países vecinos.

\section{Introducción}

La certificación de las propiedades mecánicas y eléctricas de los dispositivos FV, permite consolidar a la Universidad en el medio, y reforzar los trabajos de extensión que esta ofrece. Todo esto se ve potenciado, ya que siendo el 2017 el año de las energías renovables, se vislumbra un futuro favorable, donde los dispositivos FV comienzan a presentarse de manera más frecuente en nuestra sociedad.

Existen leyes y políticas de fomento (Ley 27.191, 2015) (Plan RenovAr, 2016) (2448/15, 2015) (M. Cáceres et al, 2012) en donde se denota una tendencia en aumento de proyectos relacionados a la generación de energía renovable, tanto de manera distribuida como autónoma. Donde se destaca el Proyecto de Energías Renovables en Mercados Rurales (PERMER) (PERMER, 2017) que brinda la posibilidad de llevar energía eléctrica hasta los usuarios rurales recónditos, mejorando su calidad de vida. En este tipo de proyectos, la importancia de realizar certificaciones de los módulos fotovoltaicos permite verificar la calidad de los componentes instalados y que se cumplan las pautas fijadas en los pliegos de licitación. De esta manera se limita el ingreso de materiales o dispositivos de baja calidad.

\footnotetext{
$\left(^{*}\right)$ Campus Deodoro Roca, Av. Libertad 5460

1. afirman@ger-unne.com.ar, FaCENA, UNNE

2.1vera@ger-unne.com.ar, FaCENA, UNNE.

3. mcaceres@ger-unne.com.ar, FaCENA, UNNE

4. bertotto.olivio@gmail.com, FaCENA, UNNE.
} 
Entre los ensayos asociados específicamente a módulos FV, se ofrecen los siguientes ensayos (IRAM 210013, 2007).

a) Inspección Visual. Según norma IRAM 210013-1

b) Curva IV y extracción de parámetros eléctricos en condiciones estándar de medida. Según norma IRAM 210013-2

c) Ensayo de aislación eléctrica. Según norma IRAM 210013-3

d) Resistencia mecánica de terminales. Según norma IRAM 210013-4

e) Ensayo de resistencia al impacto de granizo. Según norma IRAM 210013-8

\section{Metodología}

Los ensayos listados en el apartado anterior, presentan diferentes tipos de complejidad, que van desde métodos sencillos $\mathrm{y}$ rudimentarios que requieren pesas, balanzas, lupas, cronómetros, entre otros, hasta ensayos sumamente sofisticados donde se requiere equipamiento costoso y confiable, como ser; sondas de temperatura, multímetros, meghometros, celdas de referencia para determinar la irradiancia incidente, cargas electrónicas etc.

Según la complejidad del ensayo, se los puede dividir en 3 categorías principales de complejidad; baja, media y alta.

\section{Ensayos de complejidad baja.}

En este grupo de ensayos se agrupan; los de inspección visual. Los cuales requieren que un especialista busque defectos que puedan ser detectados a simple vista (o con la asistencia de una lupa) en los componentes del módulo FV, es decir, en su marco de sujeción, en el vidrio frontal, recubrimiento posterior, en las células FV, contactos eléctricos, caja de conexiones, etc. El módulo pasa la prueba si no se detectan fallas.

También en este grupo, se encuentran los ensayos de resistencia mecánica de terminales, en los cuales se debe sujetar pesas de valores tubulados sobre los contactos eléctricos del módulo FV durante tiempos determinados, el módulo pasa la prueba si la caja de conexiones o contactos no se desprenden. Las masas empleadas van en aumento progresivo hasta llegar a los $2 \mathrm{~kg}$.

\section{Ensayos de complejidad media.}

En este grupo se encuentra el ensayo de resistencia al impacto de granizo, si bien el ensayo es rudimentario, su complejidad se ve elevada debido al hecho de que se necesita disponer de un cañón de aire presurizado, para disparar municiones esféricas de hielo, de masa y temperatura definida, a una velocidad especifica según norma. Para esto, se requiere el uso de una barrera óptica, de manera de determinar la velocidad del proyectil.

En la construcción del equipo se utilizó un caño de polipropileno en donde el ingreso de aire presurizado se regula por una válvula de presión (lo que permite regular la velocidad de disparo). El sistema se acciona (dispara) por medio de una electroválvula comercial de elevado caudal, con la finalidad de ofrecer la menor resistencia posible al paso del fluido.

Los proyectiles de hielo deben ser de un diámetro uniforme de manera de obtener consistencia en los disparos. Para ello 
se empleó el uso de modelado tridimensional, por impresión 3D y posteriormente la pieza final se obtuvo por termoformado al vacío sobre las impresas en plástico de alto impacto. El diámetro de los proyectiles seleccionado de las opciones que proporciona la norma es de $25 \mathrm{~mm}$, los cuales deben ser disparados a $22,8 \mathrm{~m} / \mathrm{s}$ de velocidad (valores que deben encontrarse dentro del $+/-5 \%$ de variación) con una temperatura que ronda entre los $-5^{\circ} \mathrm{C} \mathrm{a}-15^{\circ} \mathrm{C}$.

La barrera óptica, consta de dos sistemas comerciales infrarrojos led separados una distancia determinada, donde el equipo mide el intervalo de tiempo en milisegundos entre la obstrucción de la luz de cada barrera, para ello se utilizó el quipo Pasco Scientific ME 9206A (ME 9206A. 2017).

El módulo FV supera el ensayo cuando es capaz de soportar 11 impactos distribuidos por la superficie del mismo, abarcando zonas críticas, como en las esquinas, bordes y centro del mismo.

Además en este grupo de ensayos se encuentra el de aislación eléctrica, donde por medio de una fuente continua se aplica tensión entre ambos terminales de conexión y el marco del módulo FV (o anclaje) con voltajes que van de los $500 \mathrm{~V}$ a los 1000 $\mathrm{V}+2$ veces la tensión de circuito abierto del módulo. La aceptación del ensayo ocurre si el módulo presenta una resistencia de aislación mayor a los $50 \mathrm{M} \Omega$. Para este ensayo se recurrió al equipo comercial que se dispone; medidor de aislación Fluke 1587 (Fluke 1587,2017), el cuál realiza en ensayo se manera semi-automática.

\section{Ensayo de complejidad alta.}

En este apartado se encuentra el ensayo de extraer las características eléctricas en condiciones normalizadas. Debido a la calidad, cantidad de equipamiento de medición y metodologías que se requiere para llevarlo a cabo de manera adecuada, este es el ensayo que presenta mayor grado de dificultad.

Lo primero a determinar son los coeficiente de variación de los parámetros eléctricos con la temperatura; $\alpha$, en porcentaje de variación de la corriente de cortocircuito en la temperatura y $\beta$, en porcentaje de variación de la tensión de circuito abierto con la temperatura. Estos parámetros son difíciles de determinar a sol natural ya que dependen también del valor de irradiancia $\mathrm{G}$ presente $\left(\mathrm{W} / \mathrm{m}^{2}\right)$. Debido a que difícilmente se pueden obtener intervalos de tiempo de gran amplitud térmica, en que la irradiancia se mantenga constante, se aplican modelos matemáticos para corregir su efecto. Para ello se recurre a las Ec. 1 y la Ec. 2 , entre dos estados medidos en diferentes condiciones y determinados con precisión (A. Firman et al, 2016).

$$
\begin{gathered}
I_{1}=\frac{G_{1}}{G_{2}} I_{2}\left(1+\alpha \Delta T_{c}\right) \\
V o c_{1}=V o c_{2}+\beta\left(T c_{1}-T c_{2}\right)-m \cdot \frac{k T c}{q} \cdot \ln \left(\frac{G_{2}}{G_{1}}\right)
\end{gathered}
$$

donde; $I$ es la corriente de cortocircuito $\mathrm{FV}(\mathrm{A}), \alpha$ en $\left({ }^{\circ} \mathrm{C}-1\right)$. Voc es la tensión de circuito abierto $(\mathrm{V}), \beta$ en $\left(\mathrm{V} /{ }^{\circ} \mathrm{C}\right), T c$ es la temperatura absoluta de celda $\mathrm{FV}(\mathrm{K}), m$ es el índice de idealidad del diodo, $\mathrm{k}$ es la constante de Boltzmann ( $\mathrm{J} / \mathrm{K})$, q es la car- 
ga del electrón (C) y $G$ es la irradiancia en el plano FV. En estas ecuaciones, los subíndices indican los estados determinados. De esta manera con dos estados diferentes adecuadamente determinados se puede determinar los coeficientes de variación con la temperatura.

Posteriormente, se realiza el trazado de la curva corriente vs tensión (curva $I-V$ ). Para ello se conecta una carga capacitiva electrónicamente controlada (diseñada y construida especialmente), de manera de obtener alrededor de 1000 pares de datos que van desde el cortocircuito al circuito abierto en un tiempo total de aproximadamente $100 \mathrm{~ms}$, lo que permite considerar que durante ese intervalo la irradiancia permanece constante. Para la medición de los valores de corriente y tensión se sincronizan, por medio de un protocolo especial, dos multímetros Agilent 34410A de 6 y $1 \frac{1}{2}$ dígitos (Agilent 34410A, 2017). La medición de temperatura se realiza con termocuplas calibradas, conectadas a multímetros Fluke $87 \mathrm{~V}$, calibradas en baño seco resultando una incerteza de $0,2{ }^{\circ} \mathrm{C}$. La medición de irradiancia se realiza por medio de una celda de referencia FV policristalina calibrada, cuya incerteza es del orden del $3.2 \%$, propia de los padrones secundarios de medida de este tipo. La finalidad de utilizar una celda FV de referencia de tecnología policristalina radica en el hecho de que permite considerar que posee el mismo comportamiento angular que el módulo. Además de que la misma fue calibrada en el espectro de referencia AM 1.5 (A. Firman et al, 2016).

Una vez tomada la curva se procede a realizar un ajuste matemático con el modelo que la representa, para así luego, trasladarla a las condiciones estándar de medida (STC); $1000 \mathrm{~W} / \mathrm{m}^{2}$, espectro AM 1.5 y temperatura de célula $\mathrm{FV}$ de $25{ }^{\circ} \mathrm{C}$. Una vez traslada se informan los valores de la corriente de corto circuito, la tensión de

Tabla 1. Listado de instrumentos necesarios y características de los mismos

\begin{tabular}{ll}
\hline Temperatura & $\pm 0,2{ }^{\circ} \mathrm{C}$ \\
\hline Aislación eléctrica (voltaje) & $+20 \%,-0 \%$ \\
\hline Irradiancia & $\pm 3,2 \%$ \\
\hline Tiempo & $\pm 1 \mathrm{~ms}$ \\
\hline Masa & $\pm 0,3 \mathrm{~g}$ \\
\hline
\end{tabular}

* f: indica fondo de escala. 
circuito abierto y la de su potencia nominal expresada en Watt-pico (Wp).

Para todos los casos la incerteza del instrumental utilizado se resume en la Tabla 1.

La exactitud contempla la precisión combinada de los instrumentos y métodos de medida utilizados.

\section{Resultados}

En este apartado se muestra como ejemplo, los resultados obtenidos de certificación de un módulo FV realizado en el mes de junio de 2017, con una potencia de catálogo indicada por su fabricante de $130 \mathrm{Wp}$.

Dicho módulo FV ensayado verifica satisfactoriamente las pruebas realizadas de complejidad baja y media, las cuales no requieren ser cuantificadas, solo se indica la aceptación de los ensayos de inspección visual, aislación eléctrica, resistencia mecánica de terminales y el ensayo de resistencia al impacto de granizo.

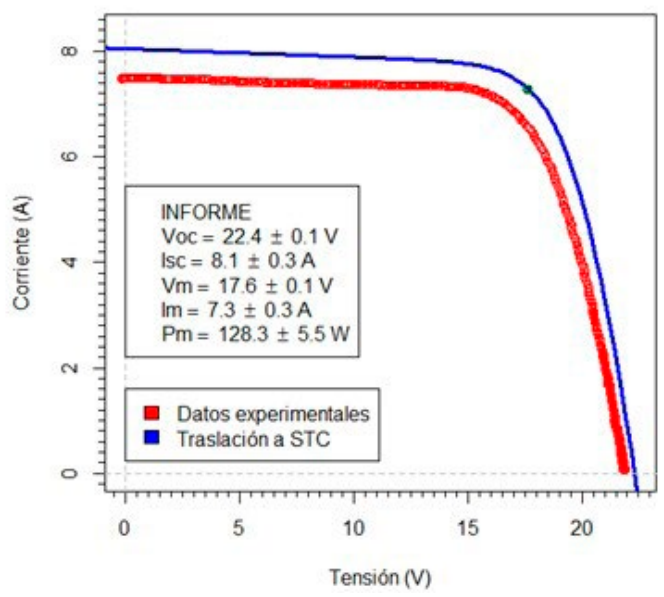

Figura 1; Curvas I-V experimental, trasladada a condiciones estándar de medida (STC) e informe de resultados.
En la Fig. 1, se muestra la curva I-V tomada experimentalmente y sobre ella la curva trasladada a condiciones estándar de medida. En el cuadro "INFORME" se indican los resultados obtenidos junto con la incerteza de la medición determinada para cada caso.

La incerteza lograda en la determinación de la potencia nominal es el 5,4\%, valor principalmente afectado por determinación de la irradiancia $(3.2 \%)$ y por la corriente que es medida a través de una resistencia tipo shunt del 0,5\%. (A. Firman et al, 2016).

\section{Conclusiones}

Se logró efectivamente adquirir, diseñar y construir todos los elementos necesarios para lograr los ensayos de certificación de módulos fotovoltaicos, según las normas IRAM vigentes en el país, las cuales fácilmente pueden ser adaptados a otros tipo de normas internacionales similares. Con lo cual se establece un laboratorio capaz de certificar ensayos.

La incerteza obtenida para la potencia nominal es del 5,4 \%, valor principalmente compuesto por la incerteza de la medida de irradiancia con celda de referencia que se encuentra en el 3,2\%, que es propio de un patrón secundario, como los utilizados normalmente en los laboratorios de la región.

Los ensayos realizados da la posibilitad de limitar el ingreso de dispositivos de baja calidad que afectarías de forma directa a los beneficiarios de los programas de promoción.

Se logró producir ensayos para distintas 
empresas del rubro. Permitiendo realizar el vínculo entre empresas nacionales y la Universidad. Aumentando la oferta de trabajos a terceros ofrecidos por la Universidad.

BIBLIOGRAFÍA.

A. Firman, L. Vera. A. Busso. (2016). Fabricación y calibración de celdas de referencia para determinar la irradiancia efectiva en SFCR. VI Congresso Brasileiro de Energía Solar - Belo Horizonte, 04 a 07 de abril de 2016.

Agilent 34410. (2017). Keysight Technologies 34410A and 34411A Multimeters Data Sheet.

Fluke 1587. (2017). 1587/1577 Insulation Multimeters, Technical data. Fluke Co.

IRAM 210013. (2007). Instituto Argentino de Normalización y Certificación. Energía solar. Módulos fotovoltaicos.

Ley 27.191. (2015). Régimen de Fomento Nacional - Uso de fuentes renovables de energía-Producción de energía eléctrica. Presidencia de la Nación.
Como segunda etapa de este trabajo se pretende la comparación de módulos calibrados localmente con otros laboratorios de países vecinos.

M. Cáceres, C. Prieb, A. Busso, L. Vera, C. Cadena. (2012). Caracterización eléctrica de inversores para sistemas fotovoltaicos conectados a la red de baja tensión. ASADES. ME 9206A. (2017). Photogate timer. Pasco Scientific.

PERMER. (2017). Proyecto de Energías Renovables en Mercados Rurales http://permer.minem.gob.ar/. Ministerio de Energía y Minería. Presidencia de la Nación.

Plan RenovAr. (2016). Plan de Energías Renovables. Ministerio de Energía y Minería. Presidencia de la nación.

S-2448/15 Ley de energía distribuida. (2015). Proyecto de ley Régimen de fomento nacional para la generación de Energía distribuida a partir de fuentes renovables. Senado de la Nación Secretaria Parlamentaria Dirección General de Publicaciones. 